\title{
Sociocultural Factors Associated with Caregiver-Psychiatrist Relationship in Taiwan
}

\author{
Hui-Ching $\mathrm{Wu}^{1}$ and Fang-pei Chen ${ }^{2} \bowtie$ \\ ${ }^{1}$ Department of Social Work, National Taiwan University, Taipei, Taiwan, ROC \\ 2Department of Social Welfare, National Chung-Cheng University, Chiayi County, Taiwan, ROC
}

Objective Research on sociocultural factors associated caregiver-provider relationship is needed to enhance family involvement in psychiatric care. This study examines from the caregiver's perspective the associations of schizophrenia attributions, stigmatization, and caregiving experiences with caregiver-psychiatrist working relationship in Taiwan.

Methods This cross-sectional study used a convenience sample of 152 Taiwanese family caregivers of persons diagnosed with schizophrenia, recruited from a grassroots organization, 4 community mental health rehabilitation centers and 2 psychiatric hospitals between July 2012 and March 2013. Multiple linear regression models were used for analysis.

Results Biological attribution was positively associated with perceived family collaboration, and so was environmental attribution with perceived informational support. Internalized stigma was negatively associated with perceived family collaboration. Caregiving rewards were positively related to both perceived family collaboration and informational support, and so was experience of problems with services to perceived family collaboration.

Conclusion The examination of family perceptions informs Western psychiatric care providers of the importance of culturally sensitive practices in developing an effective working relationship with family caregivers, particularly in regards to caregivers' casual attributions, impact of stigma, and caregiving experiences.

Psychiatry Investig 2016;13(3):288-296

Key Words Attribution, Stigma, Caregiving experience, Caregiver-provider relationship, Western psychiatry.

\section{INTRODUCTION}

Family caregivers remain an important source of care and support for people with severe mental illness in various cultural contexts. ${ }^{1,2}$ In Taiwan, there were 117,414 people with severe mental illness on the national registry ${ }^{3}$ and $83 \%$ of them lived with their family. ${ }^{4}$ Hospitals are the primary source of psychiatric care and emergency care, while community rehabilitation programs are lacking. ${ }^{5}$ Families continue to shoulder daily caregiving responsibilities and therefore could greatly benefit from effective caregiver-provider relationships.

Since the US family movement in the 70s, caregiver-provider relationship, focusing on the nature and quality of provid-

Received: February 25, 2015 Revised: May 28, 2015

Accepted: July 4, 2015 Available online: January 8, 2016

$\triangle$ Correspondence: Fang-pei Chen, $\mathrm{PhD}$

Department of Social Welfare, National Chung-Cheng University, 168 University Road, Minhsiung Township, Chiayi County 62102, Taiwan, ROC Tel: +886-5-272-0411, Fax: +886-5-272-0560, E-mail: fc10311@yahoo.com

(a) This is an Open Access article distributed under the terms of the Creative Commons Attribution Non-Commercial License (http://creativecommons.org/licenses/bync/3.0) which permits unrestricted non-commercial use, distribution, and reproduction in any medium, provided the original work is properly cited. er interactions with caregivers, has become a significant dimension in psychiatric care. ${ }^{6}$ Involving families in the provision of psychiatric care benefits both persons with mental illness and family caregivers. Education from and collaboration with mental health providers are of great importance to caregiving families. $^{7-9}$ Information about mental illness etiology and treatment, coping strategies, emotional support, community resources, respite care, and advocacy is essential. ${ }^{8}$ Structured supportive family education that provides illness information and coping strategies shows some evidence of increased knowledge and reduced burden for family caregivers. ${ }^{10}$ Chinese caregivers also benefited from family education as evidenced by significant increase in self-efficacy over a twelve-month period. ${ }^{11}$ It is thus consequential to enhance mental health providers' capacity to collaborate with family caregivers.

Co-existing with Chinese medicine and folk healing, Western psychiatry over the past decades has emerged as the primary psychiatric treatment paradigm in Taiwan. Yet little research explored caregiver-provider working relationships. In a context of plural mental health beliefs, we are interested in how caregivers' perception of working relationship might be shaped 
by their experiences with sociocultural factors and day-to-day caregiving for a family member with schizophrenia. Schizophrenia is difficult to understand and arguably one of the most stigmatized mental illnesses, ${ }^{12}$ which creates great challenges to caregivers. ${ }^{13}$ We focus on caregivers' mental illness attributions, mental illness stigmatization, and caregiving experiences that are entrenched in the sociocultural context and their associations with perceived working relationship with psychiatrists. Below we will first review the literature and then propose the theoretical framework to guide our study.

\section{Mental illness attributions}

Causal attribution of mental illness is informed by individuals' exposures to different types of mental illness etiology and sociocultural context. In Taiwan, most caregivers believed that psychosocial factors (61.2\%) were the causes of mental illness, followed by biological factors (19.0\%) and supernatural factors (15.7\%). ${ }^{14}$ Approximately $40 \%$ of people with schizophrenia and their families believed that the cause of schizophrenia was related to the supernatural phenomenon. ${ }^{15}$

Caregivers' attribution may dictate their pathways of helpseeking. ${ }^{16}$ Family caregivers who believed in destiny and supernatural causes tended to first seek help from faith healers. ${ }^{17}$ Beliefs in supernatural causes might delay or eliminate help-seeking from psychiatric treatment. ${ }^{18,19}$ Research has yet to fully examine the relationship between mental illness attribution and family-provider relationship while their relative was in care. Marshall and colleagues ${ }^{20}$ in a cross-sectional study focusing on mental illness attribution found that families were more likely to hold family causation beliefs when they had negative working experience with providers, although their beliefs in the biological causation were not associated with working experience. They suggested that families' feelings of self-blame might be reinforced by negative provider experience. ${ }^{20}$ Due to the correlational nature of the evidence, it is likely that families' beliefs in family causation prevent them from experiencing positive collaboration with providers.

\section{Mental illness stigma}

Mental illness stigma and discrimination toward people with mental illness are also experienced by family caregivers. ${ }^{21,22}$ Studies identified that the majority of families felt devalued and stigmatized due to their relative's mental illness. ${ }^{23}$ Experience of isolation and avoidance was common as a result of stigma and discrimination in all aspects of life. ${ }^{22}$

Research also documented that family caregivers likely internalize discriminatory attitudes toward themselves and suffer from self-stigma. Studies found that half of the participating caregivers reported feelings of guilt or self-blame. ${ }^{24}$ Selfstigma was associated with secrecy, ${ }^{25}$ low self-esteem, ${ }^{26}$ social withdrawal, ${ }^{27}$ and psychological distress. ${ }^{28}$

In the Chinese context families are easily subjected to both perceived and internalized stigma. Culturally, preserving "face," a metaphoric representation of dignity, is a fundamental responsibility of individuals to sustain their family's social standing. ${ }^{29}$ However, mental illness was stereotyped as unpredictable and dangerous ${ }^{30}$ and having a mental illness was often associated with debilitating capacity to fulfill expectations of adulthood and to achieve full moral standing. ${ }^{31}$ Thus having a relative with mental illness could dishonor the family name and engender shame for family caregivers. ${ }^{2}$ Shame might be further compounded by feelings of guilt and self-blame, especially for parents. ${ }^{2}$

Both perceived and internalized stigma may have impact on experiences with caregiver-provider relationship. Family caregivers are critical in facilitating help-seeking and adherence to treatments ${ }^{32}$ but they were often deterred by fear of exposure to stigma and blame from providers, friends, or other family members. ${ }^{24} \mathrm{~A}$ study revealed that persons with mental illness and family members felt that they were often not listened to, not informed, and not taken into account in the decision-making process regarding treatment, which was experienced as a form of discrimination. ${ }^{22}$

\section{Caregiving experience}

In addition to impact of mental illness stigma, caregiving for a relative with mental illness oftentimes involves profound changes in every aspect of personal life. Donnelly ${ }^{2}$ identified the theme "dancing with the rhythms of symptoms" to describe caregivers' constant struggle with managing unusual behaviors, mood fluctuations, and recurrent psychotic symptoms. Impact of these difficulties was often pervasive, and caregivers' life style might be dominated by mental illness. ${ }^{33}$ In the Taiwanese/Chinese cultural context, burden of care was associated with caregivers' psychical and mental health concerns, social constraints, and kinship strains. ${ }^{26,34-37}$ These challenges correspond to a review showing significant psychological, emotional, and medical impacts, impaired functioning, and compromised quality of life of family caregivers. ${ }^{38}$ Moreover, economic constraints and financial demands were also significant. $^{39}$ Thus far, research has mixed findings on the relation between caregiving burden and professional support. A review showed that perceived sufficiency of professional support and receiving provider-afforded behavior management advice were associated with decreased objective burden, but not overall burden. The combined instrumental and affective dimensions of professional support were not related to objective burden. ${ }^{40}$

Conversely, caregiving may be a positive experience. Research found that rewards of caregiving might be prevalent. ${ }^{41}$ 
In Chinese societies, religious beliefs such as Buddhism and cultural norms of family obligation might contribute to positive appraisal of family caregiving experiences. ${ }^{42}$ Social support may also foster caregivers' positive experience with caregiving. Perceived social support might mediate the relationship between the meaning of caregiving and caregivers' level of depression. ${ }^{43}$ Specifically, support via mental health professionals' sharing of information and collaborative interactions with family caregivers was associated with experience of caregiving gains. ${ }^{41}$ Research thus far has focused on how provider support influences caregiving experiences, and these cross-sectional studies provide evidence of associations. Likely positive caregiving experiences prompt caregivers' openness to work more closely with mental health professionals.

\section{Study framework}

Our ultimate goal of this research is to help providers of Western psychiatry understand family caregivers' perspectives in order to inform culturally sensitive practices and to enhance collaboration with family caregivers. Therefore, we focus on caregivers' appraisal of working relationship and their experiences with sociocultural influences and day-to-day caregiving on their appraisal. Similar effort has been conducted on cultural determinants of help seeking; ${ }^{44}$ a model to ensure family collaboration once in care is an important step to follow. Our study represents a preliminary effort to explore sociocultural factors associated with caregiver-provider relationship.

As shown in Figure 1, this study examines associations of schizophrenia attributions, stigmatization, and caregiving experiences with family-psychiatrist relationship. As informed by the literature, we anticipate that caregivers' endorsement of different types of attribution to schizophrenia has varied associations with experiences with family-psychiatrist relationship, operationalized by perceived family collaboration and informational support. Also we hypothesize that perceived stigma and internalized stigma are associated with perceived family collaboration and informational support. Finally, we

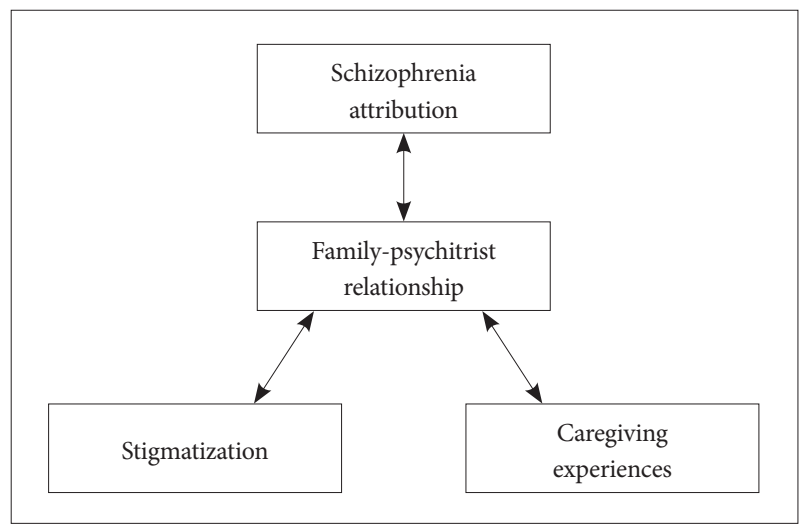

Figure 1. Study framework. expect that caregiving experiences, including experiences of problems with services, caregiving burdens, and caregiving rewards are associated with perceived family collaboration and perceived informational support.

We also include religious affiliation, household income, education, marital status, and perceived health as covariates in analysis based on literature review. Religious affiliation was found to be associated with mental illness attribution, ${ }^{45}$ mental illness stigma, ${ }^{46}$ and caregiving experience. ${ }^{42}$ Moreover, sociodemographic status, ${ }^{47}$ social support, ${ }^{48}$ and physical health ${ }^{49}$ were found to be associated with caregiving experiences. We used household income and education as proxy for sociodemographic status, marital status as proxy for social support, and perceived overall health as proxy for physical health.

\section{METHODS}

This cross-sectional study used a non-probability, convenience sample of caregivers whose relatives were diagnosed with schizophrenia in Taiwan. This research conformed to the Helsinki Declaration of 1975, as revised in 2000. The research protocol was approved by the institutional review boards at the collaborating study sites. Authors claimed no conflict of interest.

\section{Recruitment and participants}

Participants were referred to the study by senior mental health professionals from The Alliance for the Mentally Ill of R. O. C., Taiwan (TAMI), four community mental health rehabilitation centres (Zhu-Mong, De-Yang, Hsin-Hsin, and Hsing-Chiao) and two psychiatric hospitals (Kai-Shun and LaAn) between July 2012 and March 2013. To be eligible, individuals had to be 20 years old or older caring for a relative diagnosed with schizophrenia by a licensed Taiwanese psychiatrist using the criteria set forth in the Diagnostic and Statistical Manual of Mental Disorders, the 4th edition. Two hundred family caregivers were referred and informed about the study orally and in writing by the researchers. Among them, 152 caregivers provided informed written consent and completed the questionnaire. Forty-eight individuals either declined the invitation or had largely incomplete data, resulting in a response rate of $76 \%$. Verified with $\mathrm{G}^{*}$ Power version 3.0.10, ${ }^{50}$ this sample of 152 participants was able to detect an effect size of $15 \%$ increase in adjusted R-squares for the two regression models with adequate power of 0.84 , when we included 14 predictors and set the alpha level to 0.0167 to adjust for the three main hypotheses we had on attribution, stigma, and caregiving experience. 


\section{Data collection}

A Chinese self-report questionnaire was administered to participants with the assistance of a trained clinical staff member or the researchers. The participants were informed that their answers were confidential, that participation was voluntary, and that their personal data would only be seen by the researchers, who would report them in an aggregate form.

The questionnaire contained five scales: Family Collaboration Scale (FCS), Information and Advice Scale (IAS), Schizophrenia Attribution Scale (SAS), Devaluation of Consumer Families Scale (DCFS) and Experience of Caregiving Inventory (ECI). We adopted Chinese versions of DCFS and had FCS, IAS, SAS, ECI, and questions of participant background characteristics translated and back-translated between English and Chinese by the research team. Prior to data collection, the whole instrument was reviewed by the research team and Taiwanese metal health professionals to make final adjustments to meet the local use of language.

\section{Measurement}

The dependent variables, perceived family collaboration and perceived informational support, were measured by FCS and IAS, respectively. For independent variables, we used SAS to measure casual attribution, DCFS and the stigma subscale in ECI to measure perceived and internalized stigma, respectively. We used the other 9 subscales of ECI to measure the experience of caregiving including problems with services, burdens, and rewards.

\section{Family Collaboration Scale}

FCS is a four-item scale developed by Greenberg et al..$^{51}$ Participants were asked to respond to the items measuring the degree to which the psychiatrist with whom they most recently worked engaged the family as collaborators in the treatment process. The items are: 1) the staff seeks the family's opinions about the mentally ill family's situation; 2) the staff is willing to disclose information to the family about their relatives condition; 3) the staff keeps the family informed about their relatives treatment; and 4) the staff makes the family feel that they have something valuable to contribute to their relative's treatment. Participants indicated their level of agreement to each statement, ranging from strongly disagree (1) to strongly agree (4). Individual items were summed to obtain a family collaboration score. Scores ranged from 4 to 16 with a mean of 12.40 $(\mathrm{SD}=1.92)$. The Cronbach's alpha was 0.74 based on a sample of 867 participants in the United States. ${ }^{51}$ The Cronbach's alpha of the Chinese version of FCS in this study was 0.91.

\section{Information and Advice Scale}

IAS is a five-item scale developed by Greenberg et al. ${ }^{51}$ Par- ticipants were asked to respond to the items based on the information they received from the psychiatrist with whom they most recently worked. Participants indicated on a 4 -point Likert scale ( $1=$ none to $4=\mathrm{a}$ lot) the amount of information that they received from the psychiatrist regarding 5 types of information: 1) the causes, 2) medication, 3) community resources, 4) practical advice about how to cope with their mental illness, and 5) practical help in coping with their mental illness. Individual items were summed to obtain an informational support score. Scores ranged from 5 to 20 with a mean of 14.09 $(\mathrm{SD}=3.35)$. The Cronbach's alpha was 0.73 based on the sample in the study by Greenberg and colleagues. ${ }^{51}$ The Cronbach's alpha of the Chinese version of IAS in this study was 0.82 .

\section{Schizophrenia Attribution Scale}

SAS is a 19-item scale created by the researchers. We modified attribution scales used in the studies by Phillip et al. ${ }^{16}$ and Pirutinsky et al. ${ }^{52}$ based on cultural knowledge. Participants responded to the items using a 4-point Likert scale (1=very unlikely to $4=$ very likely) to indicate how likely they perceived those items to be causes of their relative's mental health condition. We conducted principal components factor analysis and identified 4 types of attribution: destiny, physical, environmental, and biological attributions. Five items (e.g., bad character and disharmony with the environment) were dropped due to poor loadings. The destiny attribution included 7 items (e.g., karma and debt to be repaid from past lives). Scores of this type of attribution ranged from 7 to 25 with a mean of 13.93 ( $\mathrm{SD}=4.73$ ). The Cronbach's alpha was 0.91 . The physical attribution included 3 items (physical injury, organ diseases, and problems during pregnancy and birth). Scores of this type of attribution ranged from 3 to 12 with a mean of 5.93 (SD= 2.12). The Cronbach's alpha was 0.77 . The environmental attribution included 2 items (upbringing and stressful circumstances in his/her life). Scores of this type of attribution ranged from 2 to 8 with a mean of $5.46(\mathrm{SD}=1.35)$. The Cronbach's alpha was 0.47 . Finally, the biological attribution included 2 items (chemical imbalance and brain injury). Scores of this type of attribution ranged from 2 to 8 with a mean of $5.57(\mathrm{SD}=$ 1.45). The Cronbach's alpha was 0.59 .

\section{Devaluation of Consumer Families Scale}

DCFS measures caregivers' perceived mental illness stigma. We adopted the Chinese version of DCFS, an 8-item scale combining the 7 items from the study by Struening et al. ${ }^{23}$ and an additional item to address the cultural concern of losing face. ${ }^{53}$ Items assess participants' perception of family-focused stigma, such as "Most people look down on families that have a member who is mentally ill living with them." Participants rated their levels of agreement to these statements using a 
4 -point scale ( 1 =strongly disagree to $4=$ strongly agree). Ratings were summed for an overall perceived stigma score. Scores ranged from 8 to 31 with a mean of $19.56(\mathrm{SD}=3.36)$. The Cronbach's alpha of the original seven-item scale was 0.71 in the study by Struening et al. ${ }^{23}$ The Cronbach's alpha in this study was 0.80 .

\section{Experience of Caregiving Inventory}

Szmukler and colleagues' Experience of Caregiving Inventory ${ }^{54}$ was employed to assess participants' caregiving experiences. The inventory has a total of 66 items, categorized into 10 subscales. Participants indicated their responses using a 5 -point scale, ranging from never (0), to almost always (4). The Cronbach's alphas of the 10 subscales ranged from 0.74 to $0.91 .^{54}$ In this study, we designated the stigma subscale as the measure of internalized stigma. The stigma subscale has 5 items such as "feeling unable to have visitors at home." Scores of this subscale ranged from 0 to 17 with a mean of $5.78(\mathrm{SD}=$ 4.03). The Cronbach's alpha was 0.76 . We devised three measures of caregiving experience, including problems with services subscale, burdens, and rewards. The problems with service subscale has 8 items, including "finding out how hospitals or mental health services work." Scores of this subscale ranged from 0 to 28 with a mean of $9.38(\mathrm{SD}=5.0)$. The Cronbach's alpha was 0.72 . The measure of burdens combined difficult behaviors, negative symptoms, effects on the family, need to backup, dependency and loss subscales, with 39 items in total. Example items included: "has difficulty looking after money" and "the illness causing a family breakup." Scores of this measure ranged from 4 to 135 with a mean of 50.17 ( $\mathrm{SD}=23.64$ ). The Cronbach's alpha was 0.93 . The measure of rewards combined positive personal experiences and good aspects of the relationship subscales, with 14 items in total. An example item is "I have learnt more about myself." Scores of this measure ranged from 3 to 53 with a mean of 27.09 ( $S D=9.91)$. The Cronbach's alpha was 0.88 .

\section{Analysis}

Descriptive analyses were conducted to describe the sample and variables. Two multiple linear regression models were constructed to analyze the associations of attributions, perceived and internalized stigma, and the 3 aspects of caregiving experience with perceived family collaboration and informational support by controlling the aforementioned covariates. Variation inflation factor was used to evaluate multicollinearity. All statistical operations were performed using SPSS 22.0.

\section{RESULTS}

\section{Sample characteristics}

Among the 152 participants $62 \%$ of them were female. The mean age of participants was $54.38(\mathrm{SD}=11.7)$ years. Among them, $71.8 \%$ were married or cohabitated, $68.6 \%$ held at least a senior high school diploma, and $25.5 \%$ lived in a low-income household. At the time of study, 46.4\% were employed, and the rest were unemployed or retired. In regards to religious affiliations, the majority (81.9\%) believed in an eastern religion (e.g., Buddhism and Taoism). About half of the participants $(52.7 \%)$ perceived themselves as being in fair health condition and $39.3 \%$ in good or better health condition. The 47 participants recruited from TAMI had no statistically significant difference in any of the characteristics and tested variables when compared with their counterpart.

According to participants' report, slightly less than one-fifth (18.7\%) of the associated ill relative had never had psychiatric hospitalization and lightly over one-fifth (22\%) had been hospitalized more than five times. In addition, about two-fifth $(42.1 \%)$ of the associated ill relatives regularly took medication, and the rest had discontinued their medication with various durations.

\section{Pairwise correlation analysis}

Table 1 shows the pairwise correlations of the variables under investigation. The correlation coefficients ranged from -0.254 to 0.61 , representing a moderate or lower level of correlation.

\section{Multiple linear regression analyses}

Table 2 shows the results of the two linear regression models. First, higher levels of perceived family collaboration were associated with higher levels of biological attribution $(\beta=0.24$, $\mathrm{p}<0.05)$, lower levels of internalized stigma $(\beta=-0.32, \mathrm{p}<0.01)$, higher levels of problems with services $(\beta=0.22, \mathrm{p}<0.05)$ and higher levels of rewards from caregiving experience $(\beta=0.28$, $\mathrm{p}<0.01)$. The full model on perceived family collaboration was statistically significant $\left(\mathrm{F}=3.12\right.$, adjusted $\left.\mathrm{R}^{2}=0.19, \mathrm{p}<0.001\right)$. Second, higher levels of perceived informational support were associated with higher levels of environmental attribution $(\beta=0.26, p<0.01)$ and higher levels of rewards from caregiving experience $(\beta=0.21, \mathrm{p}<0.05)$. The full model on perceived informational support was statistically significant $(\mathrm{F}=3.3$, adjusted $\left.\mathrm{R}^{2}=0.20, \mathrm{p}<0.001\right)$.

\section{DISCUSSION}

Results showed that participants' perceived family collaboration with, and perceived informational support from, psychiatrists were not related to their characteristics under investigation, but were variably associated with attributions, stigma, and caregiving experiences. Unlike prior research finding no association, ${ }^{20}$ our finding suggested that biological attribu- 


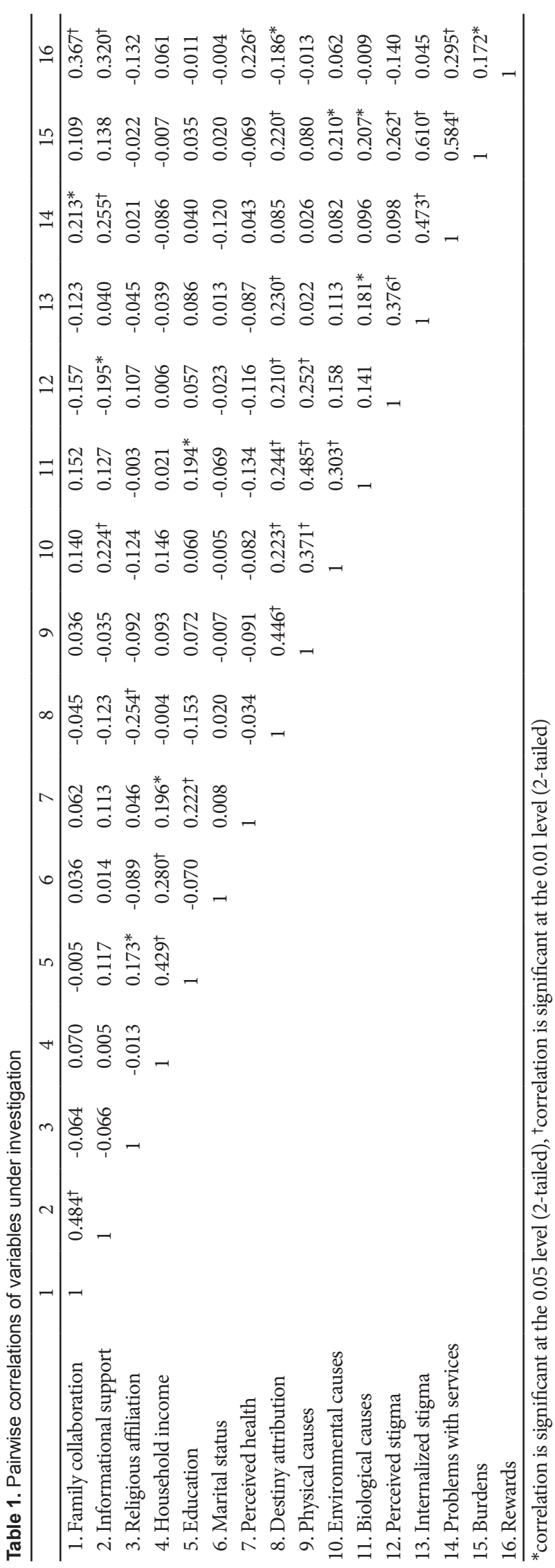

Table 2. Association of attribution, stigmatization, and caregiving experience with perceived family collaboration and informational support

\begin{tabular}{|c|c|c|}
\hline \multirow{2}{*}{$\begin{array}{l}\text { Independent } \\
\text { variables }\end{array}$} & $\begin{array}{c}\text { Family } \\
\text { collaboration }\end{array}$ & $\begin{array}{c}\text { Informational } \\
\text { support }\end{array}$ \\
\hline & \multicolumn{2}{|c|}{ Standardized beta } \\
\hline \multicolumn{3}{|l|}{ Participants' characteristics } \\
\hline Religious affiliation & -0.03 & -0.08 \\
\hline Household income & 0.08 & -0.15 \\
\hline Education & -0.02 & 0.09 \\
\hline Marital status & 0.03 & 0.04 \\
\hline Perceived health & -0.01 & 0.07 \\
\hline \multicolumn{3}{|l|}{ Attribution } \\
\hline Destiny & -0.04 & -0.16 \\
\hline Physical causes & -0.08 & -0.09 \\
\hline Environmental causes & 0.06 & $0.26^{* *}$ \\
\hline Biological causes & $0.24^{*}$ & 0.12 \\
\hline \multicolumn{3}{|l|}{ Stigma } \\
\hline Perceived stigma & 0.06 & -0.18 \\
\hline Internalized stigma & $-0.32^{* *}$ & 0.01 \\
\hline \multicolumn{3}{|l|}{ Caregiving Experience } \\
\hline Problems with services & $0.22^{*}$ & 0.17 \\
\hline Burdens & 0.12 & -0.02 \\
\hline Rewards & $0.28^{* *}$ & $0.21^{*}$ \\
\hline $\mathrm{R}^{2}$ & 0.28 & 0.29 \\
\hline Adjust $\mathrm{R}^{2}$ & 0.19 & 0.20 \\
\hline F-value & $3.12^{* * *}$ & $3.30^{* * *}$ \\
\hline
\end{tabular}

${ }^{*} \mathrm{p}<0.05,{ }^{* *} \mathrm{p}<0.01,{ }^{* * *} \mathrm{p}<0.001$

tion of schizophrenia was associated with higher levels of perceived family collaboration. This finding may indicate a promising effect on caregivers' collaboration with psychiatrists if there is a match between caregivers' mental illness attribution and the type of care in which they are involved. Moreover, our findings indicated that environmental attribution had a positive association with perceived informational support. Believing in environmental causes, such as stress from military service, school bullying, and divorced, and upbringing experiences with rigid parenting and sibling rivalry, may better justify for caregivers that schizophrenia was a resultant "breakdown," much like other diseases, which allowed them to be more open to information and advice afforded by psychiatrists in order to treat this illness. Our study did not identify significant associations of destiny or physical attributions with perceived family collaboration with and informational support from psychiatrists.

Internalized stigma, indicating caregivers' feelings of guilt and self-blame, had a strong negative association with family collaboration. This result echoes the argument by Corrigan and 
Miller. ${ }^{21}$ Caregivers who are shamed by a relative with mental illness might want to hide themselves from others and this attempt of avoidance may impede caregivers' readiness to develop collaboration with providers.

However, perceived stigma was not found to be associated with perceived family collaboration, contrary to prior research findings. ${ }^{22}$ It is likely that the scale items addressed caregivers' perceptions of general community members', rather than the psychiatrists', attitudes toward families, so they were not able to detect association with perceived family collaboration. Moreover, perceived stigma and internalized stigma were not associated with perceived informational support. The lack of association might be due to our focus on caregivers' perception of the amount of received information from psychiatrists, rather than caregivers' action in seeking information from psychiatrists, which might be more likely to be influenced by experiences of stigma.

Finally, we found positive associations of caregiving rewards with both perceived family collaboration and informational support. This finding resonates with the research conducted by Chen and Greenberg. ${ }^{41}$ Significantly, these two studies show that in both the cultural contexts of Taiwan and the US positive caregiving experience and perceived family collaboration are positively correlated. Experiences of problems with services also had a positive association with family collaboration. Possibly caregivers who had capacity of voicing issues with services might also tend to take initiative in engaging psychiatrists. Alternatively, caregivers who experienced better collaboration with psychiatrists might feel more comfortable in voicing concerns with services. Burdens, contrarily, were not found to be associated with perceived family collaboration or informational support. This finding corresponds to a prior review by Baronet. ${ }^{40}$

\section{Practice implications}

Although our study focused solely on family caregivers' perceptions on their working relationship with psychiatrists, the findings may have implications to the practice of Western psychiatric care providers in general. Findings of our study point to the significance of understanding caregivers' attribution of their relative's mental illness so that providers may use appropriate approaches to engaging caregivers. For example, Western psychiatric care providers may build upon caregivers' biological attribution and further their assistance and support for caregivers. For caregivers who believe in environmental attributions of schizophrenia, providers may adopt an emphatic approach and demonstrate acceptance of caregivers to build a relational foundation..$^{20}$ In time providers may reframe causal attributions for caregivers while introducing illness knowledge and resources in more approachable manners in order to help caregivers manage care for the ill relative.

Our findings show that internalized stigma is a strong barrier for caregivers to experience collaboration with providers. Providers' ability to recognize and help to combat caregivers' self-stigma is essential. In addition to provider-afforded education and consultation about internalized stigma, resources such as Anti-stigma Intervention ${ }^{55}$ and In Our Own VoiceFamily Companion ${ }^{56}$ also demonstrate effects in lowering internalized stigma and empower caregivers with strategies to counter effects of stigma.

More importantly, to facilitate caregivers' engagement, providers need to be critical of ways through which they may contribute to caregivers' internalized stigma. Providers need to take initiative to examine their practices that may sustain biases against caregivers or allow providers to hold onto power and resources, rather than share them with caregivers. ${ }^{57} \mathrm{Re}-$ search found providers' beliefs in family causation was significantly associated with fewer provider-family contact, ${ }^{20}$ and negative attitudes toward family members of people with mental illness were related to perceived barriers to working with families. ${ }^{7}$ Martin and Johnson ${ }^{58}$ indicated that $72 \%$ of respondents from multiple stakeholder groups believed that mental health providers should be targeted to change discriminatory attitudes and behaviors. Proper training on working with families might lead to more services provided to family, more positive attitudes toward family, and increased perceived competence about their knowledge of effective treatment for families. ${ }^{7}$

Finally, caregiver-provider relationship had a positive association with caregiving rewards. This result suggests the importance of providers' capacity to take initiative to better understand all aspects of caregiving experiences so that providers may more effectively support caregivers to cope and manage the impact of mental illness. ${ }^{20}$ Adopting narrative approaches helps to develop this holistic understanding. ${ }^{59}$ Narratives are culturally congruent and help to illustrate the psychosocial context of caregiving experiences, which provides rich insights to the feelings and meanings of caregiving. In-depth knowledge of caregiving experiences may help providers better engage families and their appreciation of caregiving experiences may become a source of empowerment.

\section{Study limitation and future study}

Our study has several limitations. First, findings had limited generalizability because of a non-probability, convenience sample. Second, the cross-sectional design was not intended to test causal relations between caregiver-psychiatrist relationship and attributions, stigma, or caregiving experiences. Rather, it was to identify a model of associations among those factors. Third, participants' responses heavily relied on recall of past experiences, which might affect data accuracy. Fourth, 
several measures of likely relevance to the caregiver-psychiatrist relationship were absent in this study. For example, length and frequency of contact with the identified psychiatrist, length of the relative's illness, and symptom severity experienced by the ill relative were possible moderators or confounders in analyzing the association of caregiver-psychiatrist relationship with attribution, stigmatization, and caregiving experience. Fifth, the translated measures (FCS, IAS, SAS, and ECI) were only tested for their face validity with Taiwanese mental health professionals. Additional work is needed to establish other dimensions of validity for these instruments. Sixth, this study relied solely on family caregivers' perceptions for all measures. To fully understand how attributions, stigma, and caregiving experience are associated with caregiver-provider relationship, future research needs to account for the providers' viewpoints as well as utilize behavioral observation to obtain objective measures of family-provider relationships. Finally, future research needs to further explore the differential associations of the domains of attributions, stigma, and caregiving experience with caregiver-provider relationship to enhance effective caregiver-provider collaboration.

\section{Conclusion}

Our study provides some evidence of varied associations of attribution, stigmatization, and caregiving experiences with caregivers' perceived family collaboration with and informational support from psychiatrists. Among four types of attributions, biological attribution was positively associated with perceived family collaboration, and environmental attribution was positively associated with perceived informational support. Internalized stigma was negatively associated with perceived family collaboration, while perceived stigma was not associated with either of the measures of family-psychiatrist relationship. Caregiving rewards were positively related to both perceived family collaboration and informational support, experience of problems with services was positively associated with perceived family collaboration, but burdens were not found to be associated with family-psychiatrist relationship. These findings from caregivers' perspectives suggest the importance of sensitivity to cultural, social, and interpersonal context of caregiver-provider interactions, and inform approaches to better engaging family caregivers in Western psychiatric care.

\section{Acknowledgments}

The authors thank The Alliance for the Mentally Ill of R. O. C., Taiwan for support of data collection. This research was funded by the National Taiwan University.

\section{REFERENCES}

1. Avasthi A. Preserve and strengthen family to promote mental health.
Indian J Psychiatry 2010;52:113-126.

2. Donnelly PL. Mental health beliefs and help seeking behaviors of Korean American parents of adult children with schizophrenia. J Multicultural Nurs Health 2005;11:23-34.

3. Ministry of Health and Welfare, R.O.C. Available at: http://www. mohw.gov.tw/cht/DOS/Statistic.aspx?f_list_no=312\&fod_list_ no=4198. Accessed September 14, 2014.

4. Department of Statistics, Ministry of the Interior, R.O.C. Physically and mentally disabled population by classification. Available at: http:// sowf.moi.gov.tw/stat/year/list.htm. Accessed September 14, 2014.

5. National Health Insurance Administration, Ministry of Health and Welfare, R.O.C. Available at: http://www.nhi.gov.tw/English/webdata/ webdata.aspx?menu=11\&menu_id=296\&WD_ID=296\&webdata id $=4456$. Accessed September 14, 2014.

6. Hatfield AB. Working collaboratively with families. Soc Work Health Care 1997;25:77-85.

7. Kim HW, Salyers MP. Attitudes and perceived barriers to working with families of persons with severe mental illness: mental health professionals perspectives. Community Ment Health J 2008;44:337-345.

8. Marshall T, Solomon P. Releasing information to families of persons with severe mental illness: a survey of NAMI members. Psychiatr Serv 2000;51:1006-1011.

9. Molinaro M, Solomon P, Mannion E, Cantwell K, Evans Jr AC. Development and implementation of family involvement standards for behavioral health provider programs. Am J Psychiatr Rehabil 2012;15:81-96.

10. Macleod SH, Elliott L, Brown R. What support can community mental health nurses deliver to carers of people diagnosed with schizophrenia? Findings from a review of the literature. Int J Nurs Stud 2011;48:100120.

11. Chan SW, Yip B, Tso S, Cheng B, Tam W. Evaluation of a psychoeducation program for Chinese clients with schizophrenia and their family caregivers. Patient Educ Couns 2009;75:67-76.

12. Marder SR, Freedman R. Learning from people with schizophrenia. Schizophr Bull 2014;40:1185-1186.

13. Caqueo-Urízar A, Gutiérrez-Maldonado J, Miranda-Castillo C. Quality of life in caregivers of patients with schizophrenia: a literature review. Health Qual Life Outcomes 2009;7:84.

14. Chang T, Song L. The correlates of psychiatric rehabilitation service use in central Taiwan. Chinese J Ment Health 1998;11:105-129.

15. Yang KY, Hsieh HC, Wu CC, Yeh TC, Chen CC. Help-seeking behavior in relatives of schizophrenics in Taiwan. Gen Hospital Psychiatry 1999; 21:303-309.

16. Phillips MR, Li Y, Stroup TS, Xin L. Causes of schizophrenia reported by patients' family members in China. Br J Psychiatry 2000;177:20-25.

17. Grover S, Nebhinani N, Chakrabarti S, Shah R, Avasthi A. Relationship between first treatment contact and supernatural beliefs in caregivers of patients with schizophrenia. East Asian Arch Psychiatry 2014;24:58-67.

18. Jorm AF. Mental health literacy. Public knowledge and beliefs about mental disorders. Br J Psychiatry 2000;177:396-401.

19. Kurihara T, Kato M, Reverger R, Tirta IG. Beliefs about causes of schizophrenia among family members: a community-based survey in Bali. Psychiatr Serv 2006;57:1795-1799.

20. Marshall T, Solomon P, Steber SA, Mannion E. Provider and family beliefs regarding the causes of severe mental illness. Psychiatr Q 2003; 74:223-236.

21. Corrigan PW, Miller FE. Shame, blame, and contamination: a review of the impact of mental illness stigma on family members. J Ment Health 2004;13:537-548.

22. González-Torres MA, Oraa R, Arístegui M, Fernández-Rivas A, Guimon J. Stigma and discrimination towards people with schizophrenia and their family members. A qualitative study with focus groups. Soc Psychiatry Psychiatr Epidemiol 2007;42:14-23.

23. Struening EL, Perlick DA, Link BG, Hellman F, Herman D, Sirey JA. Stigma as a barrier to recovery: the extent to which caregivers believe most people devalue consumers and their families. Psychiatr Serv 2001; 
52:1633-1638.

24. Angermeyer MC, Schulze B, Dietrich S. Courtesy stigma--a focus group study of relatives of schizophrenia patients. Soc Psychiatry Psychiatr Epidemiol 2003;38:593-602.

25. Phelan JC, Bromet EJ, Link BG. Psychiatric illness and family stigma. Schizophr Bull 1998;24:115-126.

26. Tsang HW, Tam PK, Chan F, Chang WM. Sources of burdens on families of individuals with mental illness. Int J Rehabil Res 2003;26:123-130.

27. Phillips MR, Pearson V, Li F, Xu M, Yang L. Stigma and expressed emotion: a study of people with schizophrenia and their family members in China. Br J Psychiatry 2002;181:488-493.

28. Martens L, Addington J. The psychological well-being of family members of individuals with schizophrenia. Soc Psychiatry Psychiatr Epidemiol 2001;36:128-133.

29. Earley PC. Face, Harmony, and Social Structure: An Analysis of Organizational Behavior Across Cultures. New York, NY: Oxford University Press; 1997.

30. Tsang H, Phidias K, Chan F, Cheung WM. Stigmatizing attitudes towards individuals with mental illness in Hong Kong. J Community Psychol 2003;31:383-396.

31. Yang LH, Kleinman A. "Face" and the embodiment of stigma in China: the cases of schizophrenia and AIDS. Soc Sci Med 2008;67:398-408.

32. Yamada A, Barrio C, Harding CM, Webster D, Atuel H, Hough RL. Cultural themes associated with pathways to initial use of psychiatric services in a tri-ethnic community sample of adults with schizophrenia. Schizophr Res 2009;115:375-376.

33. Saunders JC. Family functioning in families providing care for a family member with schizophrenia. Issues Ment Health Nurs 1999;20:95-113.

34. Huang XY, Hung BJ, Sun FK, Lin JD, Chen CC. The experiences of carers in Taiwanese culture who have long-term schizophrenia in their families: a phenomenological study. J Psychiatr Ment Health Nurs 2009; 16:874-883.

35. Cw Lam P, Ng P, Tori C. Burdens and psychological health of family caregivers of people with schizophrenia in two Chinese metropolitan cities: Hong Kong and Guangzhou. Community Ment Health J 2013; 49:841-846.

36. Liu M, Lambert CE, Lambert VA. Caregiver burden and coping patterns of Chinese parents of a child with a mental illness. Int J Ment Health Nurs 2007;16:86-95.

37. Ramsay G. Mainland Chinese family caregiver narratives in mental illness: disruption and continuity. Asian Stud Rev 2010;34:83-103.

38. Awad AG, Voruganti LN. The burden of schizophrenia on caregivers. Pharmacoeconomics 2008;26:149-162.

39. Wu EQ, Birnbaum HG, Shi L, Ball DE, Kessler RC, Moulis M, Aggarwal J. The economic burden of schizophrenia in the United States in 2002. J Clin Psychiatry 2005;66:1122-1129.

40. Baronet AM. Factors associated with caregiver burden in mental illness: a critical review of the research literature. Clin Psychol Rev 1999;19: 819-841.

41. Chen FP, Greenberg JS. A positive aspect of caregiving: the influence of social support on caregiving gains for family members of relatives with schizophrenia. Community Ment Health J 2001;40:423-435.

42. Hsiao CY, Van Riper M. Research on caregiving in Chinese families living with mental illness: a critical review. J Fam Nurs 2010;16:68-100.

43. Yen WJ, Lundeen S. The association between meaning of caregiving, perceived social support and level of depression of Taiwanese caregivers of mentally ill patients. Int J Psychiatr Nurs Res 2006;12:1378-1392.

44. Saint Arnault D. Cultural determinants of help seeking: a model for research and practice. Res Theory Nurs Pract 2009;23:259-278.

45. Teferra S, Shibre T. Perceived causes of severe mental disturbance and preferred interventions by the Borana semi-nomadic population in southern Ethiopia: a qualitative study. BMC Psychiatry 2012;12:79-87.

46. Wesselmann ED, Graziano WG. Sinful and/or possessed? Religious beliefs and mental illness stigma. J Soc Clin Psychol 2010;29:402-437.

47. Chien WT, Chan S, Morrissey J. The perceived burden among Chinese family caregivers of people with schizophrenia. J Clin Nurs 2007;16: 1151-1161.

48. Hazel NA, McDonell MG, Short RA, Berry CM, Voss WD, Rodgers MI, et al. Impact of multiple-family groups for outpatients with schizophrenia on caregivers' distress and resources. Psychiatr Serv 2004;55: 35-41.

49. Gutierrez-Maldonado J, Caqueo-Urizar A, Kavanagh DJ. Burden of care and general health in families of patients with schizophrenia. Soc Psychiatry Psychiatr Epidemiol 2005;40:899-904.

50. Fau F, Erdfelder E, Lang AG, Buchner A. G*Power 3: a flexible statistical power analysis program for the social, behavioral, and biomedical sciences. Behav Res Methods 2007;39:175-191.

51. Greenberg JS, Greenley JR, Brown R. Do mental health services reduce distress in families of people with serious mental illness? Psychiatr Rehabil J 1997;21:40-50.

52. Pirutinsky S, Rosen DD, Shapiro Safran R, Rosmarin DH. Do medical models of mental illness relate to increased or decreased stigmatization of mental illness among Orthodox Jews? J Nerv Ment Dis 2010;198: 508-512.

53. Yang LH. Expressed emotion and stigma among Chinese-Americans with schizophrenia. National Institute of Mental Health Grant K01 MH73034-01; 2005.

54. Szmukler GI, Burgess P, Herrman H, Benson A, Colusa S, Bloch S. Caring for relatives with serious mental illness: the development of the Experience of Caregiving Inventory. Soc Psychiatry Psychiatr Eidemiol 1996;31:137-148.

55. Yang LH, Lai GY, Tu M, Luo M, Wonpat-Borja A, Jackson VW, et al. A brief anti-stigma intervention for Chinese immigrant caregivers of individuals with psychosis: adaptation and initial findings. Transcult Psychiatry 2014;51:139-157.

56. Perlick DA, Nelson AH, Mattias K, Selzer J, Kalvin C, Wilber CH, et al. In our own voice-family companion: reducing self-stigma of family members of persons with serious mental illness. Psychiatr Serv 2011; 62:1456-1462.

57. McNeil S. Understanding family-centered care in the mental health system: perspectives from family members caring for relatives with mental health issues. Soc Work Meal Health 2013;11:55-74.

58. Martin N, Johnson V. A time for action: Tackling stigma and discrimination. Report to the Mental Health Commission of Canada. Available at: http://www.culturementalhealth.com/wp-content/uploads/2013/10/ Stigma_TimeforAction_MHCommission.pdf. Accessed September $14,2014$.

59. Hall JM, Powell J. Understanding the person through narrative. Nurs Res Pract 2011;2011:293837. 